\title{
Modeling Study on the Flow, Heat Transfer and Energy Conversion Characteristics of Low-Power Arc-Heated Hydrogen/Nitrogen Thrusters
}

\author{
Hai-Xing Wang • Jin-Yue Geng $\cdot$ Xi Chen • \\ Wen Xia Pan - A. B. Murphy
}

Received: 30 July 2010/Accepted: 28 September 2010/Published online: 21 October 2010

(C) Springer Science+Business Media, LLC 2010

\begin{abstract}
A modeling study is conducted to investigate the effect of hydrogen content in propellants on the plasma flow, heat transfer and energy conversion characteristics of lowpower (kW class) arc-heated hydrogen/nitrogen thrusters (arcjets). 1:0 (pure hydrogen), 3:1 (to simulate decomposed ammonia), 2:1 (to simulate decomposed hydrazine) and 0:1 (pure nitrogen) hydrogen/nitrogen mixtures are chosen as the propellants. Both the gas flow region inside the thruster nozzle and the anode-nozzle wall are included in the computational domain in order to better treat the conjugate heat transfer between the gas flow region and the solid wall region. The axial variations of the enthalpy flux, kinetic energy flux, directed kinetic-energy flux, and momentum flux, all normalized to the mass flow rate of the propellant, are used to investigate the energy conversion process inside the thruster nozzle. The modeling results show that the values of the arc voltage, the gas axial-velocity at the thruster exit, and the specific impulse of the arcjet thruster all increase with increasing hydrogen content in the propellant, but the gas temperature at the nitrogen thruster exit is significantly higher than that for other three propellants. The flow, heat transfer, and energy conversion processes taking place in the thruster nozzle have some common features for all the four propellants. The propellant is heated mainly in the nearcathode and constrictor region, accompanied with a rapid increase of the enthalpy flux, and after achieving its maximum value, the enthalpy flux decreases appreciably due to the conversion of gas internal energy into its kinetic energy in the divergent segment of the thruster nozzle. The kinetic energy flux, directed kinetic energy flux and momentum flux also increase at first due to the arc heating and the thermodynamic expansion, assume their
\end{abstract}

H.-X. Wang $(\varangle) \cdot$ J.-Y. Geng

School of Astronautics, Beijing University of Aeronautics and Astronautics, 100191 Beijing, China e-mail: whx@buaa.edu.cn

X. Chen

Department of Engineering Mechanics, Tsinghua University, 100084 Beijing, China

W. X. Pan

Institute of Mechanics, Chinese Academy of Sciences, 100190 Beijing, China

A. B. Murphy

CSIRO Materials Science and Engineering, P.O. Box 218, Lindfield, NSW 2070, Australia 
maximum inside the nozzle and then decrease gradually as the propellant flows toward the thruster exit. It is found that a large energy loss (31-52\%) occurs in the thruster nozzle due to the heat transfer to the nozzle wall and too long nozzle is not necessary. Modeling results for the NASA 1-kW class arcjet thruster with hydrogen or decomposed hydrazine as the propellant are found to compare favorably with available experimental data.

Keywords Low-power arcjet - Plasma flow and heat transfer - Numerical modeling · Hydrogen/nitrogen mixtures

\section{Introduction}

DC arc-heated subsonic-to-supersonic nozzle flow can be encountered in a variety of applications of plasma chemistry, material processing and aerospace industry, such as lowpressure plasma spraying, nano-structured film preparation using hypersonic plasma particle deposition, arc-heated thrusters, etc. The intention of this paper is to study the plasma flow, heat transfer, and energy conversion characteristics of the arc-heated nozzle flow, taking the low-power ( $\mathrm{kW}$ class) arc-heated thruster (arcjet) for aerospace application as the research object.

Many research results have been reported in recent decades about arcjet thrusters due to their already-realized and their potential space applications. In the arcjet thruster, gaseous propellant is heated by a DC electric arc struck between the cathode and the anode-nozzle (Fig. 1) to a rather high temperature (e.g. $\sim 20,000 \mathrm{~K}$ ), and the hot gas (plasma) is subsequently expanded through a Laval (convergent-divergent) nozzle, in which the internal energy of the hot gas is converted into the kinetic energy of an axially-exhausting supersonic jet and thereby produces a thrust force. Since the gaseous propellant in the arcjet thruster can be heated to a much higher temperature, the arcjet thruster may achieve a specific impulse appreciably higher than that of the conventional chemical thruster (i.e. rocket) or the resistance-heated thruster (i.e. resistojet). The plasma flow, heat transfer and energy conversion processes in the arcjet thruster are strongly coupled with the electromagnetic fields and involve many other complicated factors such as the flow transition from the subsonic to supersonic regime, electrode phenomena, plasma non-equilibrium, conjugate gas-solid heat transfer, rarefied gas effect, etc. So far our understanding on those complex processes still remains incomplete.

The arcjet thruster can be operated by use of a variety of different propellants such as hydrogen, hydrazine, ammonia, water, nitrogen, argon, etc. Usually hydrogen is a favorable propellant since the hydrogen arcjet thruster can achieve higher specific impulse. However, considering the compatibility with the existing propellant feed system of satellite launch, hydrazine is often chosen as the propellant of the arcjet thruster. In reality $2-\mathrm{kW}$ class hydrazine arcjet thrusters have been successfully applied to many satellites since the 1990s [1] for their south-north or east-west station keeping, drag compensation, etc. For such an application, hydrazine is stored as a liquid and is decomposed into a mixture of hydrogen, nitrogen, ammonia, etc. by use of a hydrazine catalyst-bed gas generator. The gaseous mixture of the decomposed hydrazine is then used as the propellant. The gas composition of the decomposed hydrazine may vary with the performance degradation and efficiency changes of the catalyst bed, while such a gas composition variation may affect the arc voltage-current characteristics and the performance parameters of the arcjet thruster. Therefore, it is desirable to clarify the effects of propellant composition on arcjet thruster characteristics. Although many modeling and experimental results concerning 


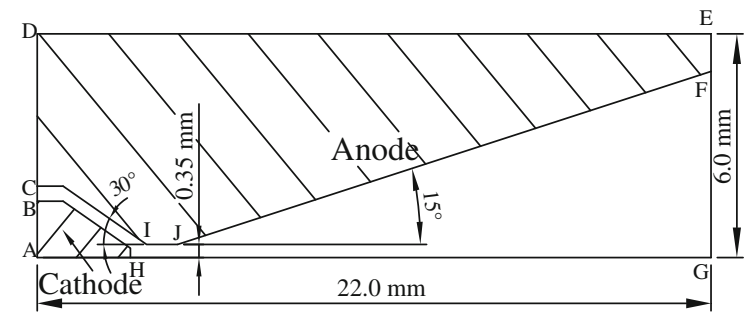

(a) Nozzle I

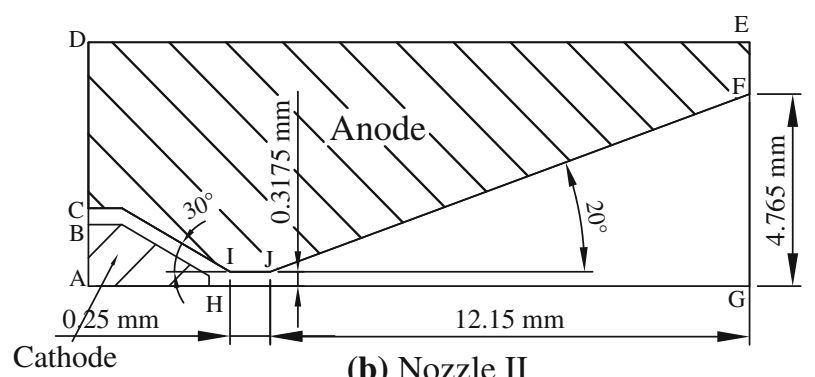

Fig. 1 Schematic diagrams of the kW-class arcjet thrusters under study. The geometrical sizes and the computational domain are also shown. a Nozzle I is the arcjet thruster designed by Institute of Mechanics of the Chinese Academy of Sciences, and b Nozzle II is that designed by NASA Lewis Center

hydrogen, nitrogen and hydrogen-nitrogen arcjet thrusters can be found in the literature, appreciably different thruster characteristics (e.g. different specific impulses, different thrust efficiencies, different arc voltages, etc.) are reported [2-19] and those results are often obtained for different power levels, different thruster construction or sizes and for different thruster operating parameters. In order to better clarify the effect of the propellant composition on the thruster characteristics, our modeling studies are performed using different kinds of the propellants but for the same thruster construction/sizes and the same operating conditions. In a previous paper we have assessed the differences between pure gases in arc-jet thrusters [20]. In this study four different propellants are considered, i.e. hydrogen, 3:1 hydrogen/nitrogen mixture to simulate completely decomposed ammonia (which will be called simulated ammonia hereafter), 2:1 hydrogen/nitrogen mixture to simulate completely decomposed hydrazine (which will be called simulated hydrazine hereafter) and nitrogen, in order to reveal the effects of the hydrogen content in the propellants on the plasma flow, heat transfer and energy conversion characteristics of the hydrogen/nitrogen arc-heated thrusters.

Modeling studies are conducted for the low-power ( $\mathrm{kW}$ class) arcjets schematically shown in Fig. 1. The construction and geometrical sizes of the arcjet thruster shown in Fig. 1a is almost the same as those used in the experimental studies of the Institute of Mechanics of the Chinese Academy of Sciences, which is called Nozzle I hereafter. The radiation-cooled arcjet thruster designed by NASA Lewis Research Center and shown in Fig. 1b has been extensively used in previous experimental and modeling studies of arcjet thrusters and has slightly different geometrical parameters from Nozzle I; it will be called Nozzle II. Nozzle II is also used in the present modeling study in order to compare the modeling prediction with the experimental data available in the literature. 


\section{Modeling Approach}

The main assumptions employed in the modeling study are as follows. (i) the gas flow in the arcjet nozzle is steady, axisymmetric, laminar and compressible; (ii) the bulk plasma is in the LTE (local thermodynamic equilibrium) state and thus the thermodynamic and transport properties of hydrogen, nitrogen or hydrogen/nitrogen mixtures are completely determined by the gas temperature and pressure [21-23]; non-LTE effects are only considered by appropriately increasing the values of gas electrical conductivity in the near-anode region, as suggested in [17, 18]; (iii) the plasma is optically thin to radiation; (iv) the azimuthal (swirling) velocity component is negligible in comparison with the axial velocity component, and (v) the flow-induced electric field is negligible in comparison with the static electric field.

Based on these assumptions, the governing equations in the cylindrical coordinate system can be written as follows [23, 24].

Mass conservation equation

$$
\frac{\partial}{\partial z}(\rho u)+\frac{1}{r} \frac{\partial}{\partial r}(r \rho v)=0
$$

Momentum conservation equations

$$
\begin{gathered}
\frac{\partial(\rho u u)}{\partial z}+\frac{1}{r} \frac{\partial(r \rho u v)}{\partial r}=-\frac{\partial p}{\partial z}+2 \frac{\partial}{\partial z}\left(\mu \frac{\partial u}{\partial z}\right)+\frac{1}{r} \frac{\partial}{\partial r}\left[r \mu\left(\frac{\partial u}{\partial r}+\frac{\partial v}{\partial z}\right)\right]+j_{r} B_{\theta} \\
\frac{\partial(\rho u v)}{\partial z}+\frac{1}{r} \frac{\partial(r \rho v v)}{\partial r}=-\frac{\partial p}{\partial r}+\frac{2}{r} \frac{\partial}{\partial r}\left(r \mu \frac{\partial v}{\partial r}\right)+\frac{\partial}{\partial z}\left[\mu\left(\frac{\partial v}{\partial z}+\frac{\partial u}{\partial r}\right)\right]-2 \mu \frac{v}{r^{2}}-j_{z} B_{\theta}
\end{gathered}
$$

Energy conservation equation

$$
\begin{aligned}
\frac{\partial(\rho u h)}{\partial z}+\frac{1}{r} \frac{\partial(\rho r v h)}{\partial r} & =\frac{\partial}{\partial z}\left(\frac{\kappa}{c_{P}} \frac{\partial h}{\partial z}\right)+\frac{1}{r} \frac{\partial}{\partial r}\left(r \frac{\kappa}{c_{P}} \frac{\partial h}{\partial r}\right)+u \frac{\partial p}{\partial z}+v \frac{\partial p}{\partial r} \\
& +\Phi+\frac{j_{z}^{2}+j_{r}^{2}}{\sigma}+\frac{5 k_{B}}{2 e}\left(\frac{j_{z}}{c_{P}} \frac{\partial h}{\partial z}+\frac{j_{r} \partial h}{c_{P} \partial r}\right)-U_{r}
\end{aligned}
$$

Electric potential (current continuity) equation

$$
\frac{1}{r} \frac{\partial}{\partial r}\left(r \sigma \frac{\partial \phi}{\partial r}\right)+\frac{\partial}{\partial z}\left(\sigma \frac{\partial \phi}{\partial z}\right)=0
$$

Here $u$ and $v$ are the axial ( $z-)$ and radial ( $r$-) components of the velocity vector $\boldsymbol{V}, p$ and $\phi$ the gas pressure and electric potential, and $k_{B}$ and $e$ are the Boltzmann constant and elementary charge, respectively. The physical properties $\rho, c_{p}, h, \mu, \kappa, \sigma$ and $U_{r}$ are the temperature- and pressure-dependent gas density, specific heat at constant pressure, specific enthalpy, viscosity, thermal conductivity, electrical conductivity and radiation power per unit volume of plasma, respectively, and are calculated for each spatial point based on local temperature and pressure by using pre-compiled LTE plasma property databases (covering the temperature range $300-30,000 \mathrm{~K}$ and pressure range $10 \mathrm{~Pa}$ to $3 \times 10^{5} \mathrm{~Pa}$ ) for each of the studied plasmas. The symbol $\Phi$ in (4) denotes the viscous dissipation term, and is calculated by

$$
\Phi=\mu\left\{2\left[\left(\frac{\partial v}{\partial r}\right)^{2}+\left(\frac{v}{r}\right)^{2}+\left(\frac{\partial u}{\partial z}\right)^{2}\right]+\left(\frac{\partial v}{\partial z}+\frac{\partial u}{\partial r}\right)^{2}-\frac{2}{3}(\nabla \cdot \boldsymbol{V})^{2}\right\}
$$


The pressure work and viscous dissipation terms have been included in the energy equation (4) since they would be non-negligible for the compressible flow in the arcjet nozzle. The Lorentz force terms have been included in the momentum equation (2) and (3) and the Joule heating rate and the electron-enthalpy transport terms have been included in the energy equation (4) in order to include the effects on the plasma flow and heat transfer of electromagnetic fields related to the DC electric arc discharge. Current density components $j_{r}$ and $j_{z}$ appearing in (2-3) and (4) are calculated by using

$$
j_{r}=-\sigma \frac{\partial \phi}{\partial r} \quad j_{z}=-\sigma \frac{\partial \phi}{\partial z}
$$

while the self-induced magnetic induction intensity $B_{\theta}$ is calculated by using

$$
B_{\theta}=\frac{\mu_{0}}{r} \int_{0}^{r} j_{z} \xi d \xi
$$

where $\mu_{0}$ is the permeability of free space.

The LTE assumption has been employed here as in most DC arc modeling studies. It is expected that non-LTE effects would exist (e.g. the electron temperature is always higher than the heavy-particle temperature in the arc-root region) and may affect the plasma flow, heat transfer and energy conversion within the arcjet thruster. A better way, of course, is to use a more comprehensive modeling approach including plasma sheaths and complete treatment of non-LTE effects, but this remains a highly challenging task due to the lack of reliable models and property data. Even the question about how to calculate the gas species composition and the thermodynamic and transport properties of two-temperature plasmas (which are a class of non-LTE plasmas) is still being seriously debated in the plasma science and technology community [25]. Although many authors attempted to use nonLTE and/or non-LCE (non-local chemical equilibrium) models in their arcjet modeling studies $[6,7,16]$, the agreement level between their predicted results and experimental data concerning the arcjet characteristic parameters (including arcjet thrust-force, specific impulse, arc voltage, gas velocity and temperature distributions, etc.) did not appreciably exceed that using a quasi-LTE model (in which non-LTE effects are only considered by appropriately increasing the values of gas electrical conductivity in the near-anode region) $[17,18]$. One possible reason is that the electrode sheaths are not included in the models, and thus the arc-root attachment at the anode-nozzle surface and the arc voltage could not be well predicted. In addition, since the gas temperatures in the downstream region of the divergent nozzle are comparatively low (e.g. gas temperature at the nozzle exit is about 2,000-3,000 K) and correspondent gas ionization degrees are quite small, the dominant gas species in the nozzle downstream would be atoms, atomic groups and/or molecules. Even if higher electron temperatures appear in this region, the bulk plasma flow and the main performance parameters of the arcjet thruster, such as the thrust force or the specific impulse (which are determined dominantly by heavy particles), would not be strongly influenced. The main problem of the employing the LTE assumption is that it leads to significantly underestimated values of the gas electrical conductivity in the near-electrode region, in which the electron temperature is expected to be significantly higher than the heavy-particle temperature [23], and thus affects the arc current flow. Refs. [17, 18] showed that using the LTE assumption for the bulk plasma flow but appropriately increasing the values of the electrical conductivity in the near-anode region to compensate the non-LTE effects can give reasonable modeling results of arcjet thruster characteristics. 
Ref. [17] used the following formula to calculate the gas electrical conductivity $\sigma_{n}$ in the near-anode region when the local gas temperature is less than $10,000 \mathrm{~K}$ :

$$
\sigma_{n}=\left(\sigma_{10000}-\sigma_{\min }\right) T / 10000+\sigma_{\min } S / m
$$

where $\sigma_{\min }$ is a defined value that can be empirically adjusted to obtain reasonable arc voltage $\left(\sigma_{\min }=10 \mathrm{~S} / \mathrm{m}\right.$ is used in [17]), and $\sigma_{10000}$ is the gas electrical conductivity at $10,000 \mathrm{~K}$ for the LTE plasma. For the region with temperatures $T$ higher than $10,000 \mathrm{~K}$, the LTE value of $\sigma$ is used. This approach is also employed in this study to treat the arcroot attachment at the inner surface of the anode nozzle.

Differently from many previous studies, the temperature distribution along the inner surface of the anode-nozzle is determined by the iterative computation process itself in this study, instead of being artificially specified $[17,18]$. To this end we employ a computational domain (see Fig. 1) that includes both the gas flow region inside the thruster nozzle and the solid wall region (anode-nozzle wall), and the energy equation (4) is solved for both the gas region and the solid region in a unified way. In the iterative solution of governing equations, the gas-phase values for $\kappa, h, c_{p}, \mu$ and $\sigma$ are used in the gaseous region, while solid-phase values of $\kappa$ and $\sigma$ but gas-phase values of $h$ and $c_{p}$ at a fixed pressure are used in the solid region. Using this approach and noting $h=\int_{T_{0}}^{T} c_{p} d T+h_{0}$ and thus $\partial h=c_{p} \partial T$, the energy equation (4) in the solid region (with zero-velocity) reduces to the following correct form:

$$
\frac{\partial}{\partial z}\left(\kappa_{s} \frac{\partial T}{\partial z}\right)+\frac{1}{r} \frac{\partial}{\partial r}\left(r \kappa_{s} \frac{\partial T}{\partial r}\right)+\frac{j_{z}^{2}+j_{r}^{2}}{\sigma_{\mathrm{s}}}+\frac{5 k_{B}}{2 e}\left(j_{z} \frac{\partial T}{\partial z}+j_{r} \frac{\partial T}{\partial r}\right)=0
$$

in which $\kappa_{s}$ and $\sigma_{\mathrm{s}}$ are the thermal conductivity and electrical conductivity of the solid material. The gas specific enthalpy $h$ and specific heat $c_{p}$ at a fixed pressure are used in the solid region only for expressing the change in local temperature arising from a change in specific enthalpy using $\partial T=\partial h / c_{p}$, and thus the energy equation in the solid region can be written in the same form as in the gas region, making it is possible to solve the energy equation for both the gas region and the solid region in a unified way. Since the values of gas specific heat $c_{p}$ at lower wall temperatures are almost independent of gas pressure, the conjugate heat transfer between the gas flow region and the solid wall region can be treated well in this manner. At the gas-solid interface region without arc-root attachment, heat flux continuity can be satisfied at the interface, as required [26]. For the gas-solid interface region with arc-root attachment, an additional source term should be included in the energy equation for the control volumes neighboring the gas-solid interface on the solid side to take into account the following additional heat transfer mechanisms: (i) the electronenthalpy flux carried by the local electron flux (or current density) (ii) the energy flux gained by electrons from the static electric field in the near-anode region and transferred to the anode wall, and (iii) the heat flux due to the electron 'condensation' (or the release of 'condensation heat') [23] at the anode wall.

Due to the axisymmetry of the thruster nozzle, only the upper half of the thruster nozzle is taken into account in the computation. The computational domain used in the modeling for both Nozzle I and Nozzle II is denoted as A-B-C-D-E-F-G-H-A in Fig. 1, in which A-B-H-A is the cathode, C-D-E-F-J-I-C is the anode-nozzle wall, whereas C-I, I-J and J-F are the inner surfaces of the convergent segment, cylindrical segment (constrictor) and divergent segment of the anode-nozzle.

The boundary conditions used in the computation are as follows. At the gas-inlet section of the arcjet nozzle (i.e. at B-C in Fig. 1), the gas stagnant pressure is set to $2.5 \mathrm{~atm}$, the gas temperature is taken to be $500 \mathrm{~K}$, the radial velocity component $v=0$ and the axial 
velocity component $u$ is calculated from the given inlet stagnant pressure $p_{O}$ and the computed local static pressure $p$ (obtained by extrapolating the static pressures at the interior grid points neighboring the inlet boundary) using the following compressible flow relation:

$$
\left(\frac{p_{0}}{p}\right)^{(\gamma-1) / \gamma}=1+\left(\frac{\gamma-1}{2}\right) \frac{u^{2}}{\gamma R T}
$$

in which $\gamma$ is the ratio of specific heats and $R$ is the ideal gas constant. The mass flow rate of incoming gas is determined by the computational process itself, as in Ref. [24].

The temperatures at the upstream boundary of the anode-nozzle (C-D) are obtained by extrapolating the temperatures at the interior grid points neighboring the upstream boundary of the anode-nozzle. On the outer surfaces D-E and E-F, the local heat flux is governed by the thermal radiation to cold surroundings $(300 \mathrm{~K})$ and an emissivity of $\varepsilon=0.3$ is used for the tungsten nozzle.

Zero velocity components are specified at all solid boundaries; axisymmetric conditions are employed along the nozzle axis; and the temperatures and velocities at the exit section of the thruster are calculated in the iteration process by extrapolating their values at the interior grid points neighboring the outlet boundary.

Zero current densities are assumed at all the boundaries except for the cathode or anode. The cathode body is included in the calculation domain, and at the rear end of cathode (A-B in Fig. 1) $u=0, v=0, T=1,000 \mathrm{~K}$ and $\partial \varphi / \partial z=I /\left(A \sigma_{c}\right)$ are used, where $I, A$ and $\sigma_{c}$ are the arc current, cathode end area, and the electrical conductivity of cathode material. $\phi=0$ is set at the outer surfaces of thruster D-E.

The governing equations are solved using a computer program [24], which is a version of the FAST-2D program [27] modified to include variable gas properties and compressible effects. The all-speed SIMPLE algorithm [24], which is incorporated into the modified FAST-2D program to simulate the subsonic-supersonic flow, is used to solve the governing equations (1-5), associated with the auxiliary relations (6-9) and (11) and the specified boundary conditions, to obtain the distributions of the velocity components, pressure and specific enthalpy (or temperature) within the whole thruster nozzle. Altogether 89 (z-direction) $\times 30$ ( $r$-direction) grid points are employed in this study, and a special numerical test shows that almost mesh-independent results have been obtained. The computer program is run using a personal computer with an Intel ${ }^{\circledR}$ Core CPU clock rate 2.66 GHz. Converged computational results are considered to be obtained when the maximum absolute value of the relative residual errors is less than $10^{-5}$ for each of the equations being solved and the computed values at a few typical grid points chosen to monitor the convergence process remain unchanged within 4 significant figures. The required run time may be as long as a few days if the computation is conducted from guessed initial fields, but the run time can be significantly shortened if the converged results for a similar case (e.g. for another propellant) are used as the initial fields and proper relaxation factors are employed. Mass conservation is ensured in the computation, i.e. the axial mass flux of the propellant is constant for all cross-sections of the nozzle.

\section{Results and Discussion}

For a fixed gas stagnant-pressure (2.5 atm) at the nozzle inlet and a constant arc current of 10 A, typical modeling results are presented in Figs. 2, 3, 4, 5, 6, 7, 8, 9 concerning the 
Fig. 2 Computed isotherms within the gas flow region and the solid wall region of Nozzle I with hydrogen (a), simulated ammonia (b), simulated hydrazine (c), and nitrogen (d) as the propellant. Isotherm interval in the gas-phase region is $1,000 \mathrm{~K}$. Inlet stagnant pressure is 2.5 atm and arc current is $10 \mathrm{~A}$ (a) Hydrogen

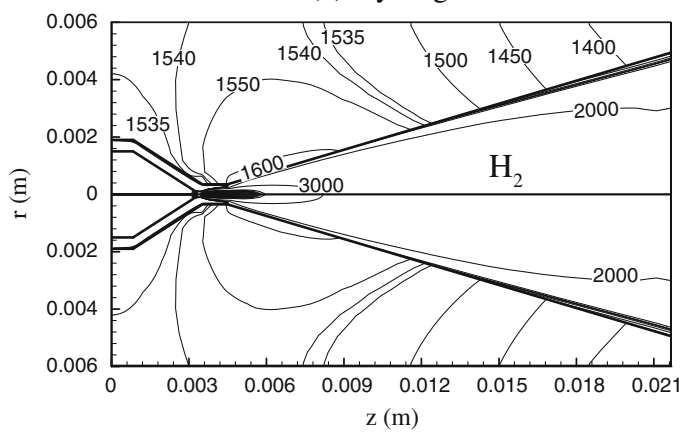

(b) Simulated Ammonia

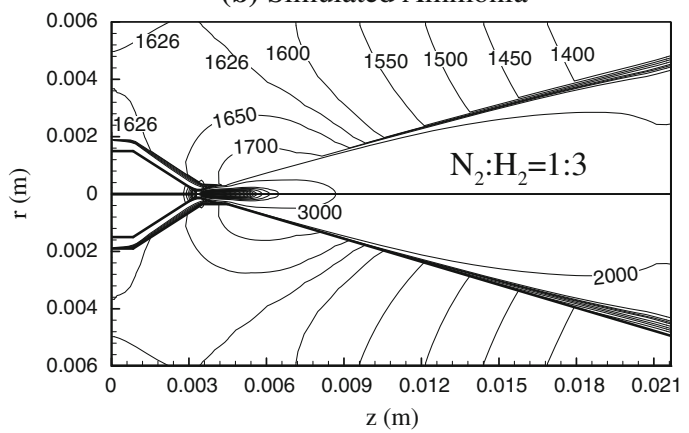

(c) Simulated Hydrazine

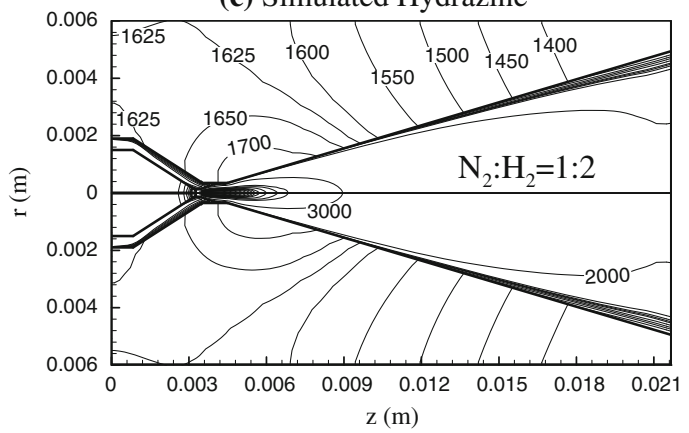

(d) Nitrogen

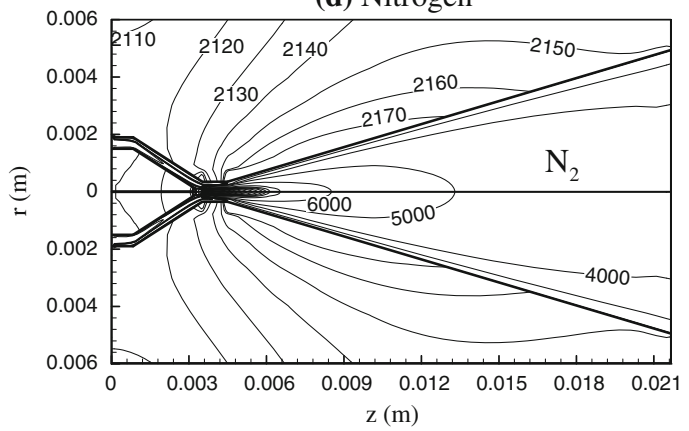




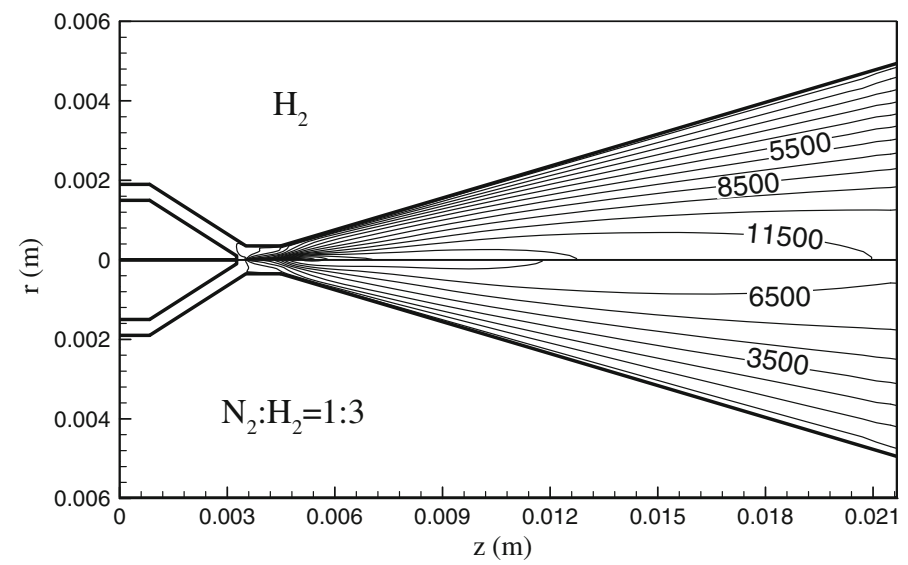

(a) Hydrogen and Simulated Ammonia

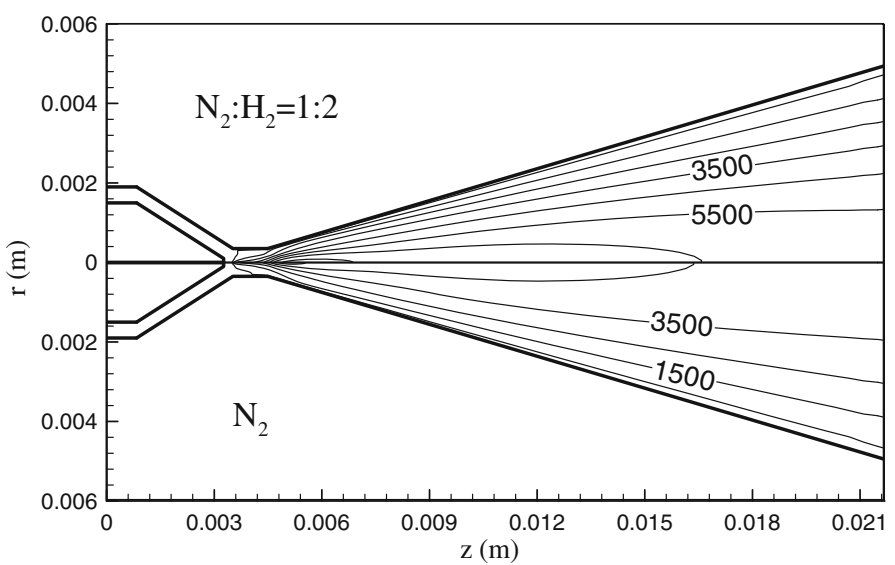

(b) Simulated Hydrazine and Nitrogen

Fig. 3 Computed axial velocity contours within the Nozzle I with hydrogen and simulated ammonia (a), as well as with simulated hydrazine and nitrogen (b), as the propellants. Isoline interval: 1,000 m/s. Inlet stagnant pressure is $2.5 \mathrm{~atm}$ and arc current is $10 \mathrm{~A}$

plasma flow, heat transfer and energy conversion characteristics of Nozzle I (see Fig. 1) using pure hydrogen, simulated ammonia, simulated hydrazine, and pure nitrogen, respectively, as the arcjet propellants.

Figures 2, 3, 4, 5 compare the computed distributions of gas temperature, axial velocity, Mach number and streamline, respectively, within the thruster nozzle for the four different propellants. Figure 2 plots the computed temperature distributions in the gas flow region and in the solid-wall region of the nozzle, showing that the temperature distributions within the thruster nozzle are similar overall for the four different propellants. The gaseous propellant entering into the thruster undergoes a rapid temperature rise in the near-cathode and constrictor region due to arc heating (Joule heating). Subsequently the heated hightemperature partially-ionized gas (plasma) expands in the diverging part of the nozzle, accompanying by an appreciable temperature decrease in the axial direction in the nozzle. There exist large radial gradients of the gas temperature in the thruster nozzle, especially in 


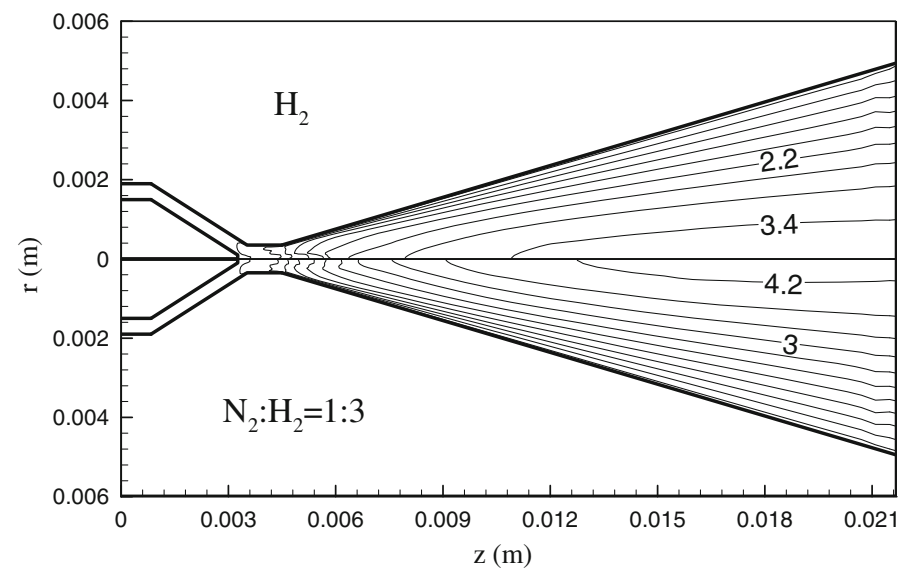

(a) Hydrogen and Simulated Ammonia

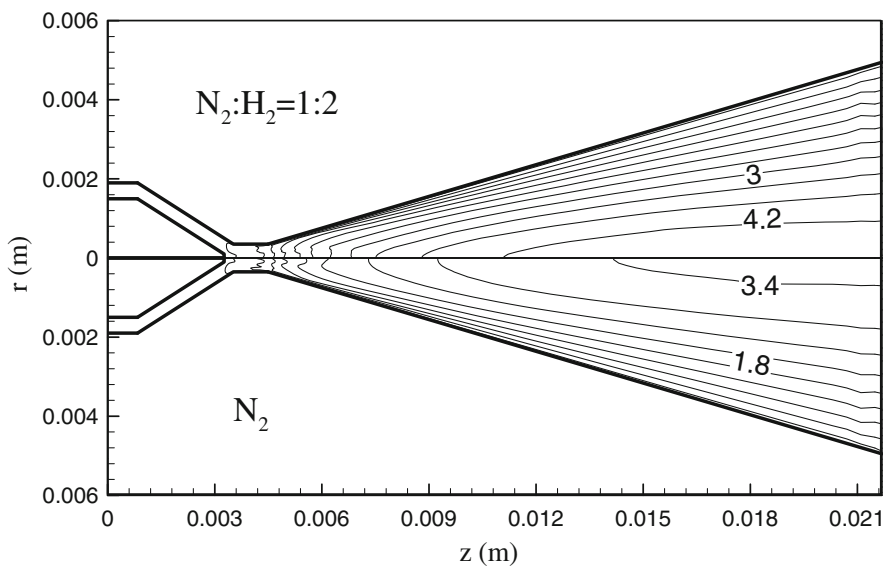

(b) Simulated Hydrazine and Nitrogen

Fig. 4 Computed Mach number contours within the Nozzle I with hydrogen and simulated ammonia (a), as well as with simulated hydrazine and nitrogen (b), as the propellants. Isoline interval: 0.4. Inlet stagnant pressure is $2.5 \mathrm{~atm}$ and arc current is $10 \mathrm{~A}$

the constrictor region and near the gas-solid interface. The temperature values in the downstream region of the nozzle and in the anode-nozzle wall for the nitrogen arcjet thruster are appreciably higher than those for the hydrogen, simulated ammonia, or simulated hydrazine arcjet thrusters. For the cases with simulated ammonia or simulated hydrazine as the propellant, the temperature values within the arcjet thruster are closer to the case with pure hydrogen than that with pure nitrogen as the propellant. The high temperature region (with gas temperature $>6,000 \mathrm{~K}$ ) appearing nearby the constrictor only occupies a small volume of the arcjet nozzle for all the four different propellants.

The computed axial velocity and Mach number distributions within the thruster nozzle are shown in Figs. 3 and 4, respectively. Overall the axial velocity or Mach number distributions are also similar for the four different propellants. Figure 3 shows that due to the conversion of the pressure energy and internal energy into the kinetic energy, the 


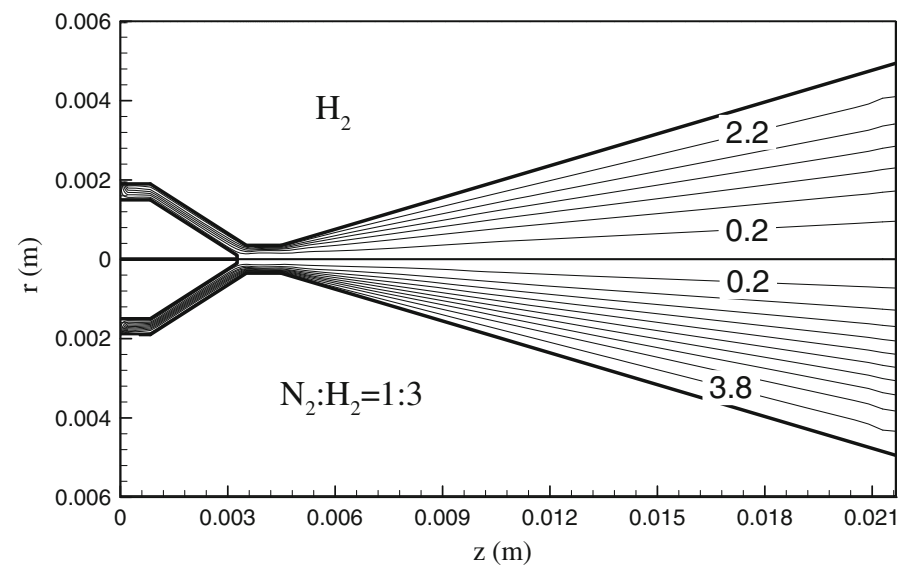

(a) Hydrogen and Simulated Ammonia

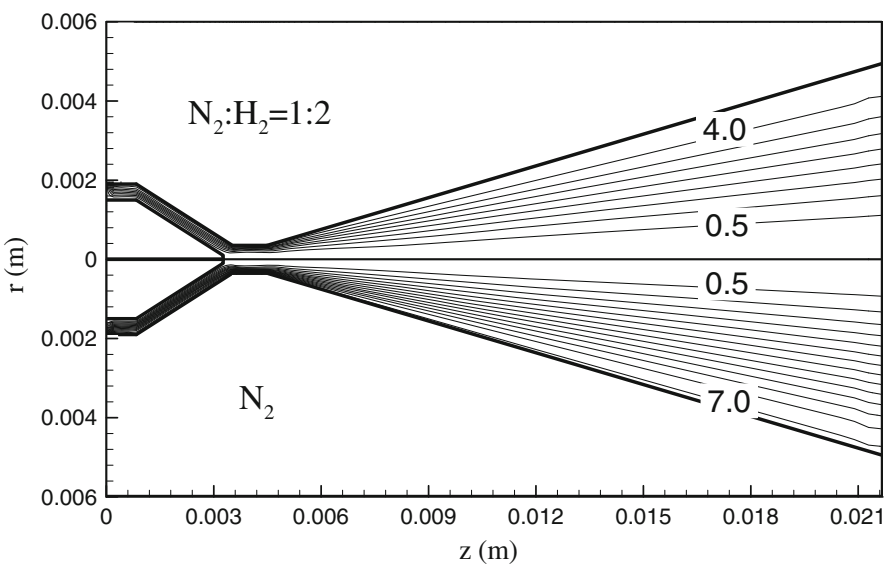

(b) Simulated Hydrazine and Nitrogen

Fig. 5 Computed streamlines within the Nozzle I with hydrogen and simulated ammonia (a), as well as with simulated hydrazine and nitrogen (b), as the propellants. Isoline interval is $0.4 \times 10^{-6} \mathrm{~kg} / \mathrm{s}$ for hydrogen and simulated ammonia, and $0.5 \times 10^{-6} \mathrm{~kg} / \mathrm{s}$ for simulated hydrazine and nitrogen. Inlet stagnant pressure is $2.5 \mathrm{~atm}$ and arc current is $10 \mathrm{~A}$

gaseous propellant flowing into the nozzle is rapidly accelerated to rather high velocities within a short axial distance. There exist large radial gradients of the axial velocity in the thruster nozzle. Unlike the conventional compressible flow in a Laval nozzle, the maximum velocity is found to appear in the interior of the arcjet nozzle (at the location near the downstream end of the constrictor) instead of at the nozzle exit. This phenomenon has been observed in experiments $[10,15]$ and is believed to be due to the complex interaction between the Joule heating, Lorentz force, viscous force and thermodynamic expansion in the constrictor and near-constrictor region. Figure 4 shows that the Mach number (i.e. the ratio of local flow velocity to local sound speed) monotonically increases in the axial direction, the flow transits from the subsonic to the supersonic regime (i.e. a transition from $\mathrm{Ma}<1$ to $\mathrm{Ma}>1$ ) within the constrictor region, and the flow becomes completely supersonic $(\mathrm{Ma}>1)$ in the diverging segment of the thruster nozzle. Since the values of 
Fig. 6 Comparisons of the computed variations of plasma temperature (a), axial velocity (b), and gas pressure (c) along the nozzle axis for Nozzle I with hydrogen, simulated ammonia, simulated hydrazine, and nitrogen as the propellants. Inlet stagnant pressure is $2.5 \mathrm{~atm}$ and arc current is $10 \mathrm{~A}$

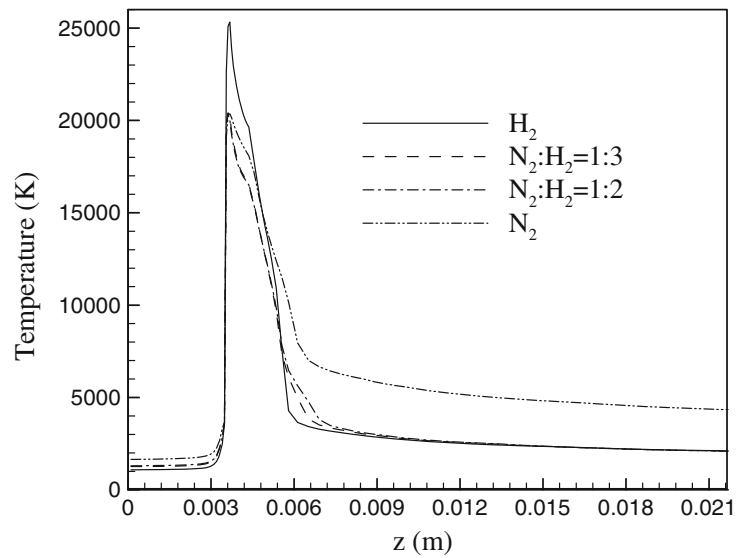

(a) Temperature variations along torch axis

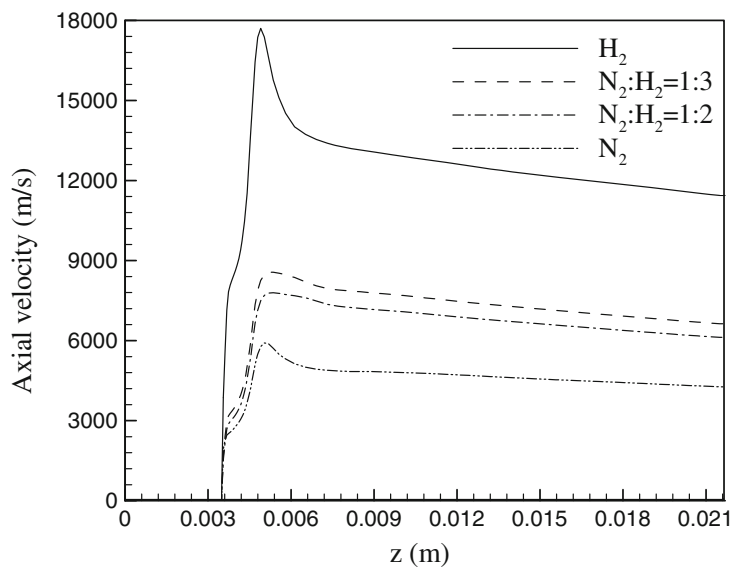

(b) Axial velocity variations along torch axis

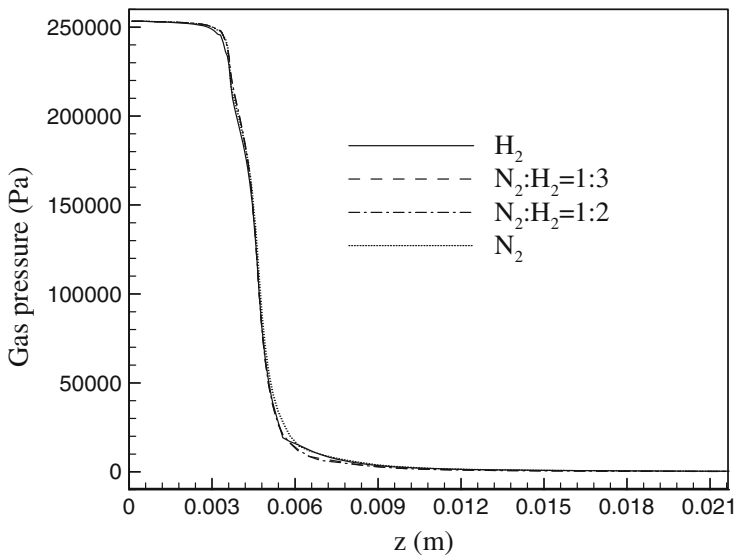

(c) Gas pressure variations along torch axis 
Fig. 7 Comparisons of computed radial distributions of the plasma temperature (a), axial velocity (b), and Mach number (c) at the thruster exit plane for Nozzle I with hydrogen, simulated ammonia, simulated hydrazine, and nitrogen as the propellants. Inlet stagnant pressure is 2.5 atm and arc current is $10 \mathrm{~A}$

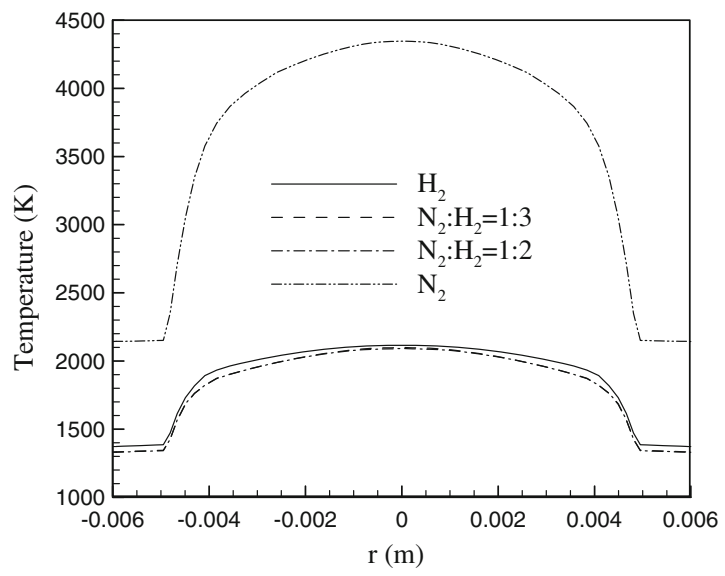

(a) Temperature profiles

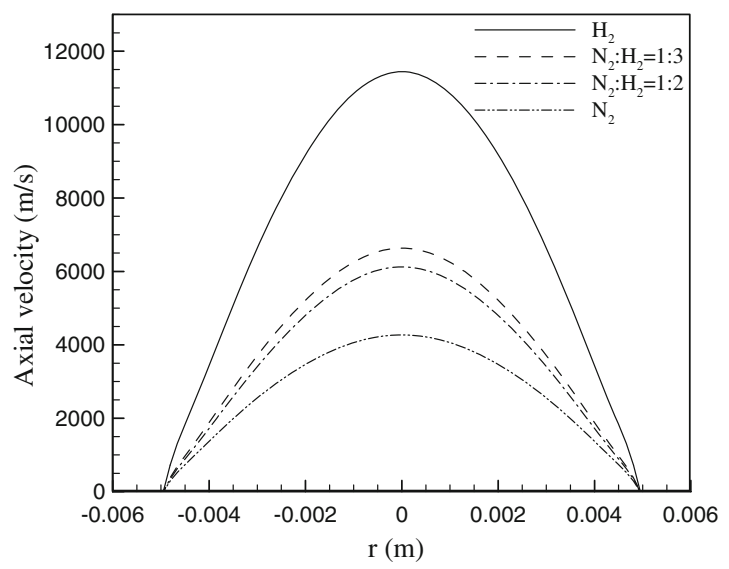

(b) Axial velocity profiles

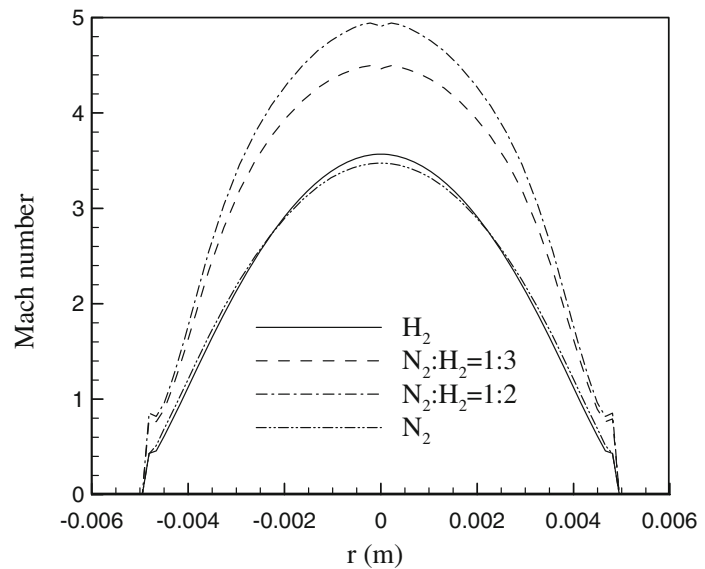

(c) Mach number profiles 
Fig. 8 Computed axial variations of the enthalpy flux (a), the kinetic energy flux (b), and the total enthalpy flux (c), normalized to the mass flow rate, for Nozzle I with hydrogen, simulated ammonia, simulated hydrazine, and nitrogen as the propellants. Inlet stagnant pressure is 2.5 atm and arc current is $10 \mathrm{~A}$

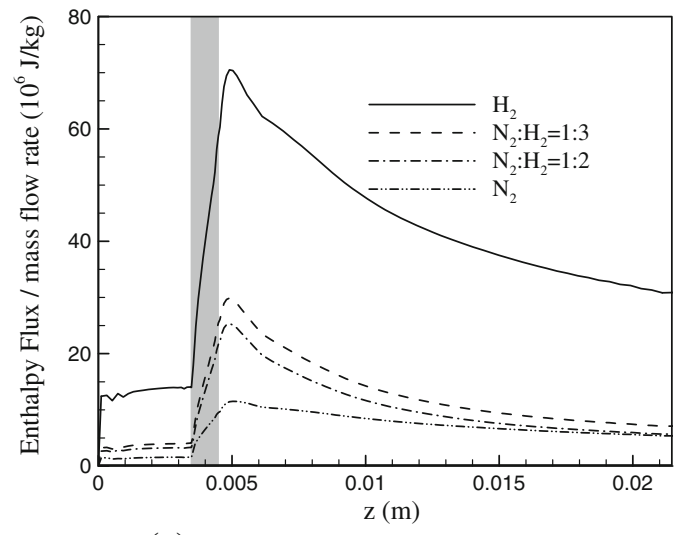

(a) Reduced enthalpy flux variations along torch axis

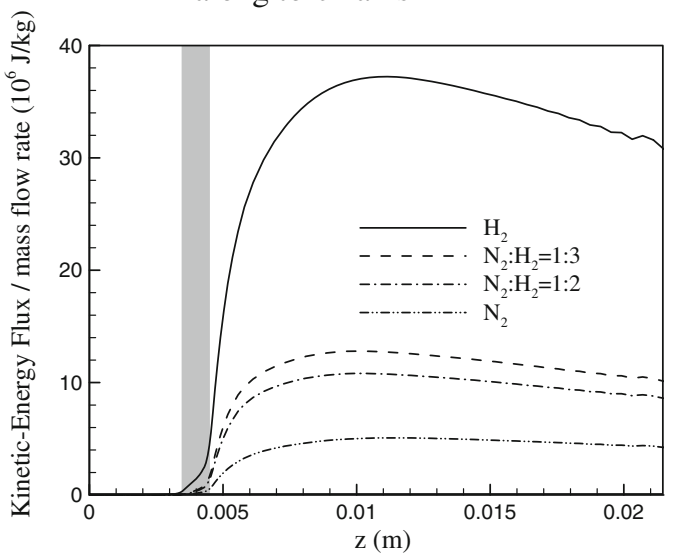

(b) Reduced Kinetic energy flux variations along torch axis

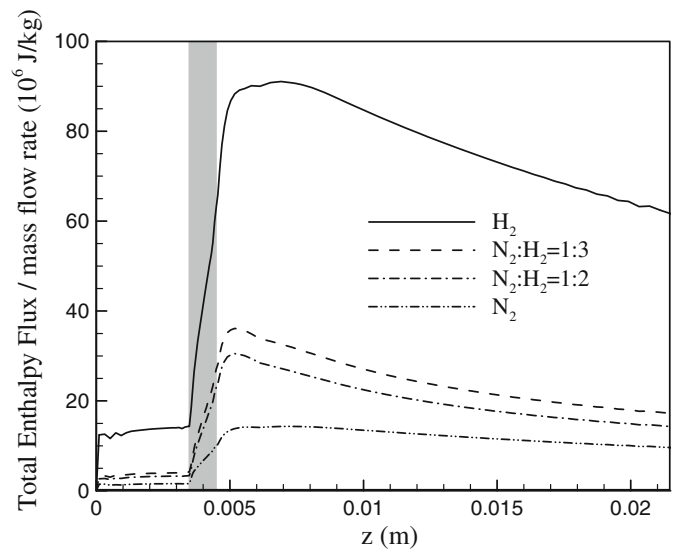

(c) Reduced total enthalpy flux variations along torch axis 
Fig. 9 Computed axial variations of the directed kineticenergy flux (a) and the momentum flux (b), normalized to the mass flow rate, for Nozzle I with hydrogen, simulated ammonia, simulated hydrazine, and nitrogen as the propellants. Inlet stagnant pressure is $2.5 \mathrm{~atm}$ and arc current is $10 \mathrm{~A}$

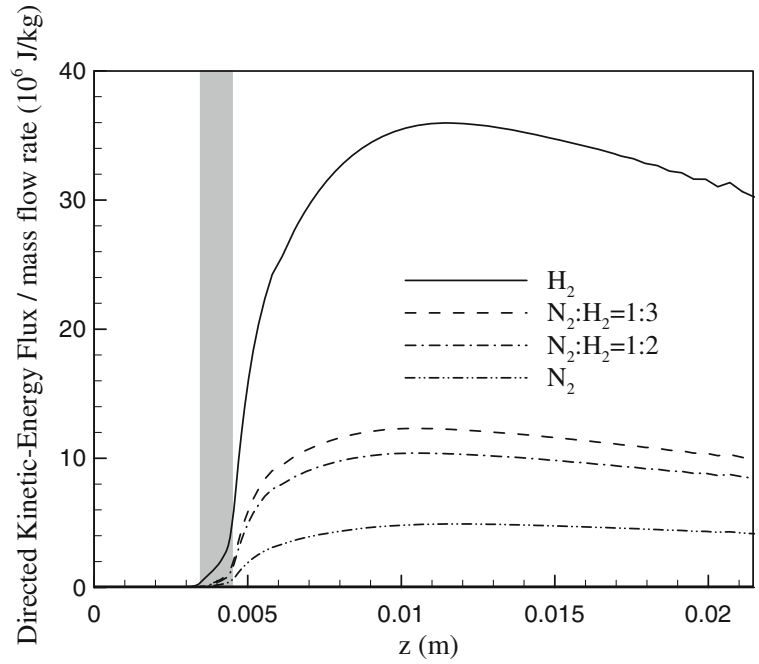

(a) Reduced directed Kinetic-energy flux variations along torch axis



(b) Reduced momentum flux variations along torch axis

thermodynamic and transport properties of the simulated ammonia and the simulated hydrazine fall into the range between their counterparts of the pure hydrogen and pure nitrogen, the predicted axial velocity for a given location within the arcjet nozzle increases in the order nitrogen, simulated hydrazine, simulated ammonia, and hydrogen, i.e. increases with decreasing mean molecular weight of the propellant, as seen in Fig. 3. Figure 4 shows that the differences in the computed distributions of the Mach number between the hydrogen, simulated ammonia, simulated hydrazine and nitrogen arcjet thrusters are comparatively small in comparison with those in the computed velocity distributions. This is because the sound speed also depends on the molecular weight of the 
gas, and the sound speed also increases in the order nitrogen, simulated hydrazine, simulated ammonia and hydrogen. It is noted that unlike the predicted velocity, the predicted Mach number for a given location within the arcjet nozzle presented in Fig. 4 for hydrogen is close to that for nitrogen, but the predicted Mach number for simulated ammonia or simulated hydrazine is appreciably larger than that for hydrogen or nitrogen.

Figure 5 shows that the computed streamline distributions within the thruster nozzle are generally similar, but that different mass flow rates are obtained for the four different propellants. The predicted streamline distributions demonstrate that most of the incoming gaseous propellant flows towards the nozzle exit through the outer cooler region near the anode-nozzle wall. Since the inner-surface temperatures of the anode-nozzle wall directly affect the gas temperatures and velocities in the near-wall region and thus affect the thrust force and specific impulse of the arcjet thruster, accurate calculation of the temperature distribution along the inner-surface of the thruster nozzle is important. This is the reason why in this study the anode-nozzle wall is included in the computational domain and the energy equation is solved in a unified way for both the gas and solid regions.

Figures $6 \mathrm{a}-\mathrm{c}$ compare the computed variations along the nozzle axis of the plasma temperature, axial velocity and pressure, respectively, for the hydrogen, simulated ammonia, simulated hydrazine and nitrogen arcjet thrusters. Figure 6a clearly shows that the on-axis temperature increases very rapidly at first due to the arc heating in the nearcathode and constrictor region, assumes its maximum value near the downstream end of the constrictor region, and then decreases quite rapidly to comparatively low values as the hot gas expands in the divergent segment of the nozzle. The highest plasma temperatures appearing in the rapid-heating region are 25,236, 20,409, 20,349, and 20,300 K, respectively, for the hydrogen, simulated ammonia, simulated hydrazine, and nitrogen arcjet thrusters. Among the four different propellants, the predicted maximum temperature is lower for the nitrogen arcjet, but the temperature decay rate in the supersonic segment of the nitrogen arcjet nozzle is appreciably lower so that a relatively high temperature appears at the exit of the nitrogen arcjet. Corresponding computed values of the arc voltage obtained in this modeling study are 110.2, 82.0, 77.3, and $51.1 \mathrm{~V}$, respectively, for the four different propellants, i.e. the highest arc voltage $U$ is predicted for the hydrogen arcjet thruster, and the lowest $U$ for the nitrogen arcjet thruster, with the same inlet stagnantpressure $(2.5 \mathrm{~atm})$ and the same arc current $(10 \mathrm{~A})$ for the case studied. Hence, for this situation appreciably more electric power would be required to supply the hydrogen arcjet thruster than the simulated ammonia, simulated hydrazine, or nitrogen arcjet thrusters. Ref. [11] described an experiment conducted to compare the voltage-current characteristics of arcjet thrusters with simulated ammonia and simulated hydrazine as the propellants, and showed that for the case with the same mass flow rate (not the same inlet stagnant-pressure as assumed here) and arc current, the arc voltage for the simulated ammonia thruster was about 10 volts higher than that for the simulated hydrazine thruster. Similar results to those in [11] were also mentioned in [28]. The reason is believed to be that hydrogen extracts energy more effectively from the arc due to its high thermal conductivity, and thus the propellant with higher hydrogen content requires a larger electric field intensity (or voltage gradient) to sustain the arc with a constant current. The axial velocity distributions along the thruster axis shown in Fig. 6b are somewhat different from the on-axis temperature distributions shown in Fig. 6a. The axial velocity increases rapidly at first until a maximum is achieved, and then decreases gradually as the propellant flows towards the thruster exit. The axial velocity assumes its maximum value at the axial location about $0.55 \mathrm{~mm}$ downstream of the constrictor as a result of the complex interaction between the Joule heating, Lorentz force, viscous force and thermodynamic expansion. The fact that the 
highest axial velocity always appears inside the nozzle instead of at the nozzle exit is a point of difference between the arcjet thruster and the conventional compressible flow in a Laval nozzle, as mentioned above. In the divergent segment of the thruster nozzle, the axial velocities at the nozzle axis decrease from the maximum values of 17,659, 8,563, 7,794, and 5,911 to $11,440,6,633,6,123$, and $4,269 \mathrm{~m} / \mathrm{s}$ at the thruster exit, respectively, for the hydrogen, simulated ammonia, simulated hydrazine, and nitrogen arcjet thrusters. For the hydrogen arcjet thruster, the predicted axial variation of axial velocity is consistent with the measurements reported in Refs. $[10,15]$. Figure $6 \mathrm{c}$ shows that the axial variations of gas pressure along the nozzle axis are almost the same for the four different propellants, and that the rapid decrease of the gas pressure occurs in the constrictor region.

Figure 7 compares the computed radial distributions of the gas temperature (a), axial velocity (b) and Mach number (c) at the nozzle exit plane (F-G in Fig. 1). As seen in Fig. 7a, the predicted highest temperatures at the centre of thruster exit plane are 2,115, 2,099, 2,091, and 4,346 K, respectively, for the hydrogen, simulated ammonia, simulated hydrazine, and nitrogen arcjet thrusters, i.e. the nitrogen arcjet thruster assumes a much higher exit temperature. The temperature distribution in the arcjet thruster exit plane for simulated ammonia or simulated hydrazine as the propellant is close to that of the hydrogen arcjet thruster. Figure $7 \mathrm{~b}$ and $\mathrm{c}$ show that the predicted maximum axial velocities at the nozzle exit are 11,440,6,633, 6,123, and 4,269 m/s, while the highest Mach numbers are $3.568,4.465,4.911$, and 3.474 , respectively, for the hydrogen, simulated ammonia, simulated hydrazine, and nitrogen arcjet thrusters. Although the magnitudes of the axial velocity at the exit of arcjet thruster are quite different for the four propellants due to their different molecular weights (with a much larger exit velocity for the hydrogen arcjet thruster), the difference in their Mach number distributions is comparatively small because the gas sound speed also depends on the molecular weight of the gas, as indicated above. Figure 7c also shows that the highest Mach numbers at the nozzle exit for simulated ammonia and simulated hydrazine are appreciably larger that those for hydrogen and nitrogen arcjet thrusters.

After converged results have been obtained, the axial mass flux (or mass flow rate) $G$ for a given propellant remains unchanged for all cross-sections along the nozzle axis and can be calculated by $G=\int_{0}^{r_{0}}(\rho u) 2 \pi r d r$ at the nozzle exit section. The specific impulse $I_{s p}$ of the arcjet thruster (defined as the thrust force per unit weight flow rate of propellant, with $s$ as the unit) can be calculated by $I_{s p}=F /(G g)=\int_{0}^{r_{0}}\left(\rho u^{2}\right) 2 \pi r d r /(G g)$ at the nozzle exit section (if the gas pressure at the nozzle exit is equal to that in the vacuum chamber). Here $r_{O}$ is the radius of the nozzle exit, $F$ is the axial momentum flux at the thruster exit (or the thrust force) and $g$ is the gravitational acceleration. For the case with inlet stagnant pressure of $2.5 \mathrm{~atm}$ and arc current of $10 \mathrm{~A}$, the computed mass flow rates are 15.3, 25.2, 27.6 , and $44.4 \mathrm{mg} / \mathrm{s}$, the computed arc voltages are $110.2,82.0,77.3$, and $51.1 \mathrm{~V}$ (excluding the sheath voltage drops), the computed thrust forces are 112.1, 105.5, 106.6, and $120.2 \mathrm{mN}$, whereas the computed specific impulses are 748, 427, 394, and $276 \mathrm{~s}$, respectively, for the hydrogen, simulated ammonia, simulated hydrazine and nitrogen arcjet thrusters.

Since the energy conversion within the thruster nozzle determines the performance of arcjet thruster, it would be useful to analyze the effect of gas composition on the energy conversion characteristics. In order to better compare the energy conversion characteristics of different propellants, a reduced enthalpy flux, $E_{h}$, a reduced kinetic energy flux, $E_{k}$, a reduced directed kinetic-energy flux, $E_{k a}$, and a reduced momentum flux, $E_{m}$, are introduced in this study to characterize the energy conversion processes. They are defined as the 
local enthalpy flux, kinetic energy flux, directed kinetic-energy flux and momentum flux across a cross-section of the nozzle, normalized to the mass flow rate of the propellant flowing in the nozzle, i.e.

$$
\begin{gathered}
E_{h}=\int_{0}^{r_{z}}(\rho u h) 2 \pi r d r / G \\
E_{k}=\int_{0}^{r_{z}} \rho u\left[\frac{1}{2}\left(u^{2}+v^{2}\right)\right] 2 \pi r d r / G \\
E_{k a}=\int_{0}^{r_{z}} \rho u\left(\frac{1}{2} u^{2}\right) 2 \pi r d r / G
\end{gathered}
$$

and

$$
E_{m}=\int_{0}^{r_{z}} \rho u^{2} 2 \pi r d r / G
$$

Here $r_{z}$ is the radius of the nozzle cross-section at the axial location $z$. For the case with fixed inlet stagnant pressure and arc current, since the mass flow rate $G$ remains constant in the whole thruster nozzle for a given propellant but its values are different for different propellants, using the reduced quantities $E_{h}, E_{k a}, E_{k}$, and $E_{m}$ (normalized to the mass flow rate) is more suitable to compare the energy conversion characteristics for different propellants. Equations (12-15) can also be regarded as the expressions for the local mass-fluxweighted averages of the specific enthalpy $(h)$, the specific kinetic energy $\left(\left(u^{2}+v^{2}\right) / 2\right)$, the specific directed kinetic-energy $\left(u^{2} / 2\right)$, and the specific momentum, i.e. the axial velocity $(u)$, respectively, at a nozzle cross-section, and all the reduced quantities $E_{h}, E_{k}$, $E_{k a}$, and $E_{m}$ depend on the axial position $z$.

Figure 8 compares the computed variations of the reduced enthalpy flux $\left(E_{h}\right)$, the reduced kinetic energy flux $\left(E_{k}\right)$, and the reduced total enthalpy flux $\left(E_{h}+E_{k}\right)$, while Fig. 9 compares the computed variations of the reduced directed kinetic energy-flux $\left(E_{k a}\right)$ and the reduced momentum flux $\left(E_{m}\right)$ along the nozzle axis for the four propellants. The shadow in Figs. 8 and 9 represents the constrictor region in the arcjet nozzle. It is seen from Fig. 8a that the reduced enthalpy flux of the propellant increases rapidly in the nearcathode and constrictor region due to the arc heating, similarly to the on-axis temperature rise shown in Fig. 6a. After the maximum value has been achieved, the reduced enthalpy flux decreases gradually in the axial direction due to the thermodynamic expansion of propellant in the supersonic nozzle, but its decay rate is appreciably less than that of the onaxis temperature shown in Fig. 6a, reflecting the effect of heat and momentum transfer in the radial direction in the nozzle. After arc heating is completed, the value of the reduced enthalpy flux for a given axial location increases with increasing hydrogen content in the propellant, assuming much higher values for hydrogen than the other three propellants. The decay rate of the reduced enthalpy flux of hydrogen, simulated ammonia, or simulated hydrazine is somewhat more rapid than that of nitrogen propellant, apparently due to the high thermal conductivity of the hydrogen component in the propellants. Figure $8 \mathrm{~b}$ shows that the reduced kinetic energy flux increases rapidly at first due to the gas heating and the 
thermodynamic expansion in the supersonic nozzle and then decreases gradually as the propellant flows towards the nozzle exit due to the energy loss caused by the heat transfer to the nozzle wall and the effect of viscous drag. The reduced kinetic energy flux reaches its maximum value in the interior of the nozzle, increases with increasing hydrogen content in the propellant, and assumes a much higher value for the hydrogen than the other three propellants for a given axial location. Figure 8c shows that for each studied propellant, the reduced total enthalpy flux first increases rapidly due to the electric energy input, and after achieving its maximum value it decreases due to heat transfer to the nozzle wall. The difference between the maximum value and the exit value represents the energy loss, which is mainly due to heat transfer from the plasma flow to the nozzle wall. For a given axial location, the value of reduced total enthalpy flux increases with increasing hydrogen content in the propellant. It is very interesting to note that the energy loss due to the heat transfer to the nozzle wall is considerable. The values of the reduced total enthalpy flux $\left(E_{h}+E_{k}\right)$ decrease from the maximum values $91.0,36.1,30.5$, and $13.9 \mathrm{MJ} / \mathrm{kg}$ to the exit values $61.7,17.2,14.3$, and $9.6 \mathrm{MJ} / \mathrm{kg}$, respectively, for the hydrogen, simulated ammonia, simulated hydrazine, and nitrogen arcjet thrusters, i.e. the total-enthalpy-flux loss occurring in the divergent segment of the Laval nozzle due to the heat transfer to the nozzle wall can be as large as about $31 \%$ (for hydrogen or nitrogen) to about $52 \%$ (for simulated ammonia and simulated hydrazine) of the maximum total enthalpy flux. This finding is not consistent with the previous viewpoint that the lower thrust efficiency (35-42\%) of arcjets is mainly due to frozen flow loss while the energy loss due to the heat transfer to the nozzle wall is comparatively small $(\sim 15 \%)$ [9]. The percentages of the total enthalpy-flux loss (31 or $52 \%$ ) have been evaluated with respect to the maximum value of the total enthalpy flux in the nozzle instead of to the actual electric power (the product of arc current and actual arc voltage) supplied to the arcjet thruster, since the actual arc voltage cannot be accurately predicted by the model without a full treatment of the plasma sheaths. The percentage will be somewhat smaller if the total enthalpy-flux loss is evaluated with respect to the actual electric power supplied to the arcjet, but the predicted heat transfer loss would still be appreciably larger than that the previous estimated value (heat transfer loss $\sim 15 \%$ ) if an additional sheath voltage as large as $\sim 25 \mathrm{~V}$ were included in the power evaluation. The difference in the evaluated percentages of heat transfer loss is perhaps a consequence of the improved treatment of conjugate gas-solid heat transfer that has been adopted in this modeling study.

The directed kinetic-energy flux is the energy flux directly related to the production of thrust force. Figure 9a shows that the reduced directed kinetic-energy flux for a given axial position is only slightly less than corresponding reduced kinetic-energy flux shown in Fig. 8 b. This is because the radial velocity component in the nozzle is appreciably lower than the axial velocity component, and thus the gas kinetic energy is determined dominantly by the axial velocity component. Figure $9 \mathrm{~b}$ shows that the reduced momentum flux achieves its maximum value in the interior of the nozzle, implying that too long a nozzle is not helpful in obtaining a high specific impulse for the studied case. Similarly to the results in Fig. 8, both the local reduced directed kinetic-energy flux and the local reduced momentum flux increase with increasing hydrogen content in the propellant and assume much higher values for the hydrogen than the other three propellants for a given axial location.

Many experimental results $[10,12,14,15,19,30-34]$ have been reported in the literature for the $\mathrm{kW}$-class arcjet thruster designed by the NASA Lewis Research Center (shown in Fig. 1b as Nozzle II) and using hydrogen or simulated hydrazine as the propellant. Slightly differently from Nozzle I, Nozzle II has a 0.635 -mm-diameter constrictor, a diverging-nozzle with half-angle of 20 degrees and an exit/constrictor area ratio of 225 
$[31,32]$. We have also performed a modeling study of Nozzle II in order to compare our modeling results with the experimental data available in the literature.

For the case with hydrogen as the propellant, experimental results have been reported by Cappelli and his co-workers [10, 15, 30-34]. Our predicted results for the axial-velocity variation along the nozzle axis and the radial profiles of the axial velocity and gas temperature at the exit plane of Nozzle II are thus compared with their experimental data. Figure 10a compares the predicted axial-velocity variation along the nozzle axis with the experimental data presented in [32]. In the experiment, the mass flow rate of hydrogen propellant was $14.2 \mathrm{mg} / \mathrm{s}$, the input electric power was $1.4 \mathrm{~kW}$, and the velocity measurements were performed using the laser-induced fluorescence (LIF) method. The measured axial velocity decreases monotonically along the nozzle axis from the peak value of approximately $17.5 \mathrm{~km} / \mathrm{s}$ appearing at the axial location $1.3 \mathrm{~mm}$ downstream from the constrictor to around $12.0 \mathrm{~km} / \mathrm{s}$ at the thruster exit [32]. It can be seen from Fig. 10a that predicted axial variation of the axial velocity along the nozzle axis agrees well with the corresponding experimental data. Figure $10 \mathrm{~b}$ compare the predicted radial distribution of the axial velocity at the arcjet nozzle exit with corresponding experimental results presented in [30] for case with hydrogen flow rate of $13 \mathrm{mg} / \mathrm{s}$ and the electric power of $1.48 \mathrm{~kW}$ (arc voltage and current are $139 \mathrm{~V}$ and $10.3 \mathrm{~A}$ ). It is seen that the agreement between the experimental and predicted results is fair in the central part of the jet, while the discrepancy becomes appreciable in the fringe region, probably due to the existence of rarefaction effects near the anode-nozzle wall. Figure 10c compares the predicted radial distribution of the gas temperature at the exit section of the Nozzle II with the corresponding experimental data presented in [31]. The experimental data in Fig. 10c represent the translational temperatures measured using the LIF method $0.4 \mathrm{~mm}$ downstream of the arcjet exit plane for the case with thruster power of $1.43 \mathrm{~kW}$ and $\mathrm{H}_{2}$ mass flow rate of $13 \mathrm{mg} / \mathrm{s}$. As shown in Fig. 10c, the predicted radial profile of gas temperature at the arcjet exit represented by continuous line is reasonably consistent with the LIF measured data after the Stark-broadening correction is included [31]. For the Nozzle II with hydrogen mass flow rate of $15.2 \mathrm{mg} / \mathrm{s}$ and arc current of $10 \mathrm{~A}$, our predicted arc voltage is $110.3 \mathrm{~V}$ (the sheath voltage is not included) and the predicted specific impulse is $798 \mathrm{~s}$. For the case with hydrogen mass flow rate of $13 \mathrm{mg} / \mathrm{s}$ and arc current of and $10.3 \mathrm{~A}$, the measured arc voltage was $139 \mathrm{~V}[30,34]$, while the measured specific impulse was $830 \mathrm{~s}$ as mentioned in [34]. The reason that the predicted voltage is about 21-29 V lower than the measured data of [30] and [34] is that the plasma sheath has not been fully treated in the modeling study. The predicted specific impulse is also lower than the measured value, but doesn't seem possible to attribute this difference to the use of a quasi-LTE model, since an even lower specific impulse would be predicted if non-LTE effects were included. As expected, the present quasi-LTE model cannot correctly predict the electron number density at the nozzle exit with comparatively low gas temperatures. If LTE is assumed and the Saha equation is used to calculate the electron densities at the exit gas pressure and temperatures, the calculated values of electron density would be more than 10 orders of magnitude lower than the measured values $\left(10^{19}-10^{20} \mathrm{~m}^{-3}[31,33]\right)$. However, the presence of electrons at densities of $10^{19}-10^{20} \mathrm{~m}^{-3}$ will have little influence on the arcjet thrust, since the gas ionization degree at the arcjet exit is very small.

On the other hand, for the case with the simulated hydrazine as the propellant, a spatially resolved time-of-flight (TOF) electrostatic probe method was used in Refs. $[14,29]$ to measure the radial profiles of the plasma axial-velocity at the exit plane of Nozzle II with a propellant mass flow rate of $50 \mathrm{mg} / \mathrm{s}$. For the case with arc current of $10 \mathrm{~A}$, the measured arc voltage and the specific impulse of arcjet thruster were $112 \mathrm{~V}$ and 
Fig. 10 Comparisons of the computed results and the experimental data concerning the axial-velocity variations along the nozzle axis (a), the radial profiles of the axial velocity at the exit plane (b), and the radial profiles of gas temperature at the exit plane (c), for Nozzle II with hydrogen as the propellant. Arc current is $10 \mathrm{~A}$

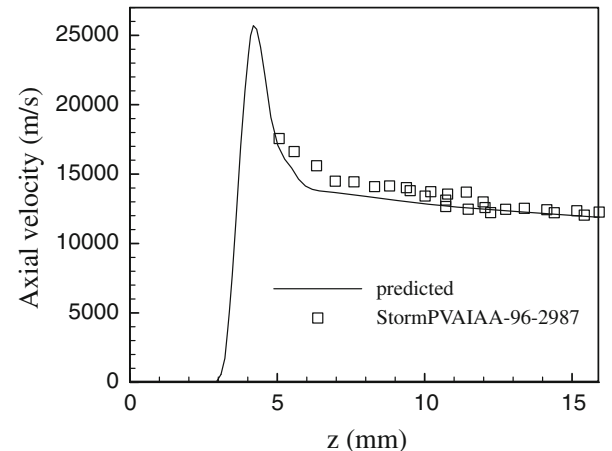

(a) Comparison of predicted axial velocity variation along nozzle axis with the experimental results of Storm et al [32]

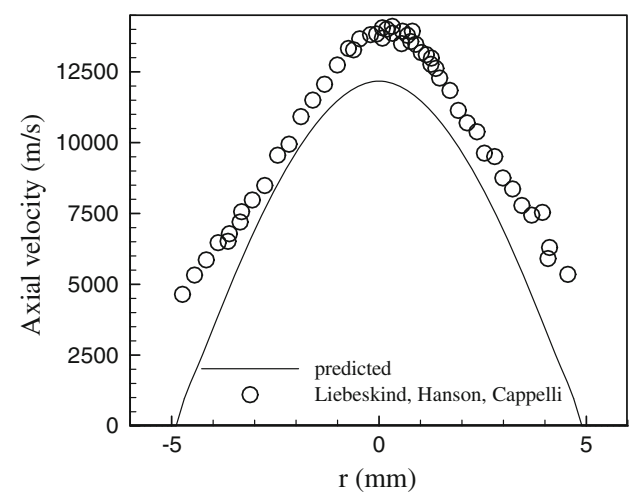

(b) Comparison of predicted radial profile of axial velocity at the exit plane with the experimental results of Liebeskind et al [30]

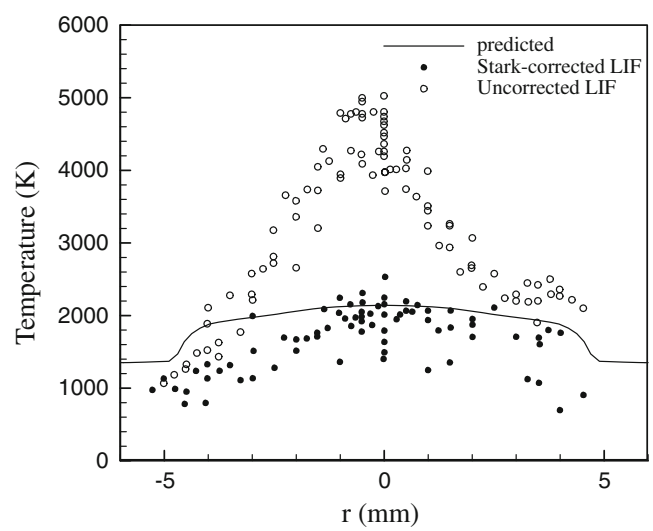

(c) Comparison of predicted radial profile of gas temperature at the exit plane with the experimental results of Storm et al [31] 
$420 \mathrm{~s}$, respectively. Corresponding modeling results for the case with mass flow rate of $50.1 \mathrm{mg} / \mathrm{s}$ and arc current of $10 \mathrm{~A}$ are $90.7 \mathrm{~V}$ (the sheath voltage is not included) for the arc voltage and $412 \mathrm{~s}$ for the specific impulse. Figure 11a compares our predicted radial profile of the axial velocity at the nozzle exit with the TOF probe results obtained in Refs. $[14,29]$. The predicted values of the axial velocity agree well with the measured data, although the predicted radial profile of the axial velocity is somewhat narrower than the measured profile. The narrower radial profile is expected because the modeling results are presented at the nozzle exit, while the TOF probe measurement was conducted in the nearexit plume. In addition, the rarefied gas effect is not considered in the modeling. The $\mathrm{N}_{2}$ vibrational and rotational temperatures measured by Zube and Myers [5] are shown in Fig. 11b for Nozzle II with simulated hydrazine as propellant, mass flow rate of $47.6 \mathrm{mg} / \mathrm{s}$, and arc current of $9 \mathrm{~A}$. The operating conditions used in their experiments are somewhat different from ours (the mass flow rate is $50.1 \mathrm{mg} / \mathrm{s}$, and the arc current is $10 \mathrm{~A}$ ), but the predicted centerline temperatures still agree reasonably well with the experimental data.

Although LTE is assumed, with the only non-LTE effect included being an increased gas electrical conductivity in the near-anode region, the modeling predictions are reasonably consistent with available experimental results for hydrogen and simulated hydrazine arcjet thrusters. The quasi-LTE model would also be useful for the study of many other complicated problems, including how to better treat the arc-root attachment, to clarify the rarefied gas effect in the downstream low-pressure region of nozzle flow, to reveal the demixing effect in the gas mixture, etc. However, the predicted results of the quasi-LTE model regarding the arc voltage and arc-root attachment are not yet satisfactory. Further improvement of the model is required in subsequent studies. On the other hand, considering the non-equilibrium phenomena in the arcjet thruster is extremely important if one is interested in the parameters dominated by non-LTE and/or non-LCE effects, such as the electron temperature, the number density of electrons or other ionized or excited species, etc. As mentioned above, in the literature there have been many papers studying non-equilibrium phenomena in arcjet thrusters. Even a non-LCE and four-temperature (different translational, rotational, vibrational and electron temperatures) model has been used in a recent study of the arc-heated non-equilibrium flow [35]. So far the non-equilibrium studies still suffer from the lack of reliable models and property data and cannot properly treat the plasma sheaths. Further research efforts are needed to clarify how nonequilibrium phenomena affect the arcjet thruster characteristics.

\section{Conclusions}

Numerical simulations have been carried out to study the effect of hydrogen content in hydrogen-nitrogen propellants on the plasma flow, heat transfer and energy conversion characteristics of low-power ( $\mathrm{kW}$ class) arcjet thrusters. With hydrogen, simulated ammonia, simulated hydrazine and nitrogen as the propellants, the modeling results were compared for the same thruster construction and operating parameters (inlet stagnant pressure and arc current). The axial variations of local enthalpy flux, kinetic-energy flux, total enthalpy flux, directed-kinetic-energy flux and momentum flux normalized to the mass flow rate have been used to analyze the energy conversion process within the thruster nozzle. The modeling results show that with the increase of the hydrogen content in the propellants, the predicted axial velocity at the thruster exit, arc voltage, and specific impulse of the arcjet thruster increase, but the gas temperature at the nitrogen thruster exit is significantly higher than that for other three propellants. The total-enthalpy-flux loss due 
Fig. 11 Comparisons of the computed results and the experimental data concerning the radial profiles of the axial velocity at the exit plane (a) and the axial variations of gas temperature along the nozzle axis (b), for Nozzle II with simulated hydrazine as the propellant. Arc current is $10 \mathrm{~A}$

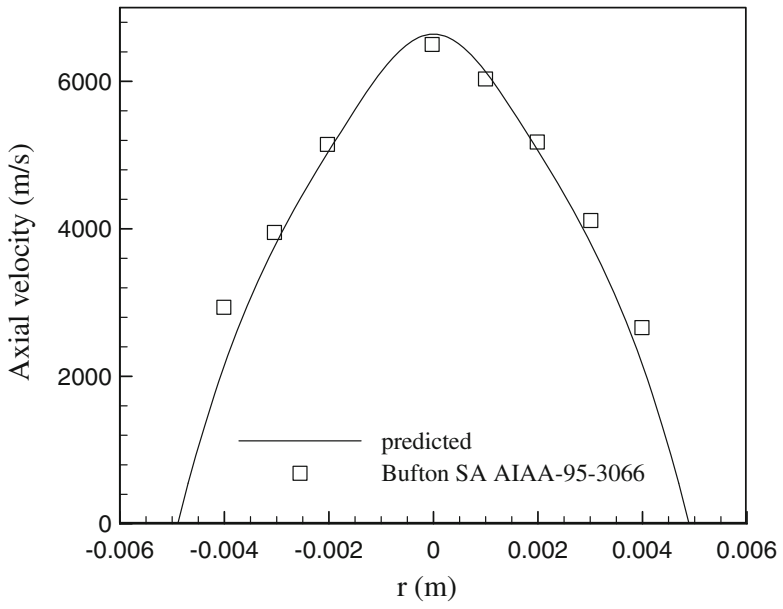

(a) Comparison of predicted radial profile of axial velocity at the exit plane with the experimental results of Bufton et al [29]

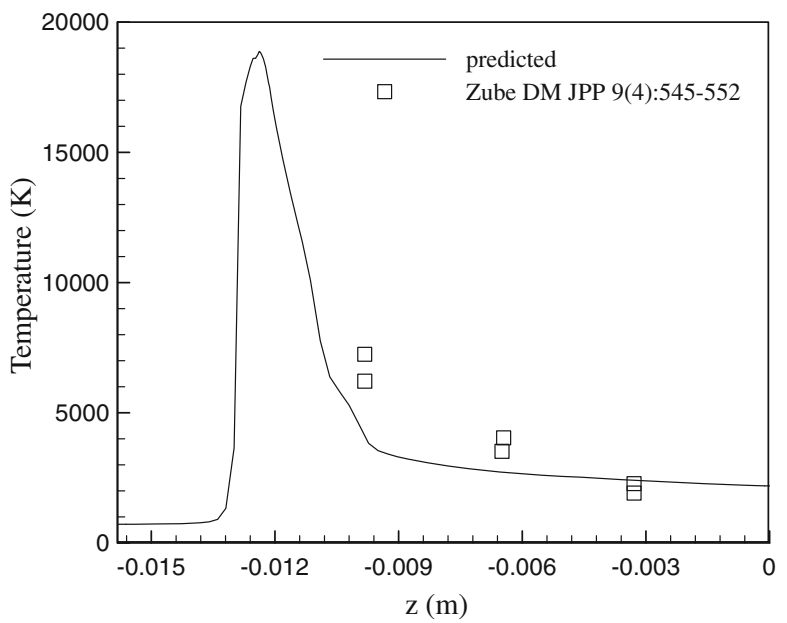

(b) Comparison of predicted gas temperature variation along nozzle axis with the experimental results of Zube et al [5]

to the heat transfer from the plasma flow to the nozzle wall is found to be as large as $\sim 31 \%$ of the maximum total enthalpy flux for hydrogen or nitrogen thrusters and $\sim 52 \%$ for simulated ammonia or simulated hydrazine thrusters. The computed reduced momentum flux assumes its maximum value inside the nozzle, implying that a somewhat shorter nozzle can be used for the studied case. Numerical simulation of the NASA 1-kW class arcjet thruster with hydrogen or hydrazine as the propellant was also performed, and the predicted results for the variation of gas temperature and axial velocity along the nozzle axis and for the radial distributions of the axial velocity and temperature at the thruster exit agree reasonably well with the corresponding experimental results. 
Acknowledgments This work was supported by the National Natural Science Foundation of China (Grant Nos. 50836007, 10775164, 11072020).

\section{References}

1. Sackheim RL (2006) Overview of United States space propulsion technology and associated space transportation systems. J Propuls Power 22:1310-1333

2. John RR, Bennett S, Connor JF (1963) Arcjet engine performance: experiment and theory. AIAA J $1: 2517-2525$

3. Sankovic JM, Curran FM (1991) Arcjet thermal characteristics. AIAA Paper No. 91-2456

4. Curran FM, Haag TW (1992) Extended life and performance test of a low-power arcjet. J Spacecr Rocket 29:444-452

5. Zube DM, Myers RM (1993) Thermal nonequilibrium in a low-power arcjet nozzle. J Propuls Power 9:545-552

6. Miller SA, Martinez-Sanchez M (1996) Two-fluid nonequilibrium simulation of hydrogen arcjet thrusters. J Propuls Power 12:112-119

7. Megli TW, Krier H, Burton RL (1996) Plasmadynamics modeling for nonequilibrium processes in $\mathrm{N}_{2} / \mathrm{H}_{2}$ arcjets. J Thermophys Heat Transf 10:554-562

8. Lichon PG, Sankovic JM (1996) Development and demonstration of a 600-Second mission-average $\mathrm{I}_{\mathrm{sp}}$ arcjet. J Propuls Power 12:1018-1025

9. Butler GW, Cassady RJ (1996) Directions for arcjet technology development. J Propuls Power 12:1026-1034

10. Cappelli MA, Storm PV (1996) Interior plasma diagnostics of arcjet thrusters. J Propuls Power 12:1070-1076

11. Auweter-Kurtz M, Glocker B, Gölz T, Kurtz HL, Messerschmid EW, Riehle M, Zube DM (1996) Arcjet thruster development. J Propuls Power 12:1077-1083

12. Burton RL, Bufton SA (1996) Exit-plane electrostatic probe measurements of a low-power arcjet. J Propuls Power 12:1099-1106

13. Burtner D, Keefer D, Ruyten W (1996) Low-power ammonia arcjet: numerical simulations and laserinduced fluorescence measurements. J Propuls Power 12:1123-1128

14. Bufton SA, Burton RL (1997) Velocity and temperature measurements in a low-power hydrogen arcjet. J Propuls Power 14:768-774

15. Storm PV, Cappelli MA (1998) Arcjet nozzle flow-field characterization by laser-induced fluorescence. Appl Opt 37:486-495

16. Aithal SM, Subramaniam VV, Babu V (1999) Comparisons between numerical model and experiments for a direct current plasma flow. Plasma Chem Plasma Process 19:487-504

17. Rhodes RP, Keefer D (1990) Numerical modeling of an arcjet thruster. AIAA Paper No. 90-2614

18. Butler GW, King DQ (1992) Single and two fluid simulations of arcjet performance. AIAA Paper No. 92-3104

19. Butler GW, Boyd ID, Cappelli MA (1995) Non-equilibrium flow phenomena in low power hydrogen Arcjets, AIAA Paper No. 95-2819

20. Wang HX, Chen X, Pan WX, Murphy AB, Geng JY, Jia SX (2010) Modelling study to compare the flow and heat transfer characteristics of low-power hydrogen, nitrogen and argon arc-heated thrusters. Plasma Sci Technol 12 (in press)

21. Murphy AB, Arundell CJ (1994) Transport coefficients of argon, nitrogen, oxygen, argon-nitrogen, and argon-oxygen plasmas. Plasma Chem Plasma Process 14:451-490

22. Murphy AB (2000) Transport coefficients of hydrogen and argon-hydrogen plasmas. Plasma Chem Plasma Process 20:279-297

23. Chen X (2009) Heat transfer and fluid flow under thermal plasma conditions, chap 2. Science Press, Beijing (in Chinese)

24. Han P, Chen X (2001) Modeling of the subsonic-supersonic flow and heat transfer in a DC arc plasma torch. Plasma Chem Plasma Process 21:249-264

25. Rat V, Murphy AB, Aubreton J, Elchinger MF, Fauchais P (2008) Treatment of non-equilibrium phenomena in thermal plasma flows. J Phys D Appl Phys 41:183001

26. Chen X, Han P (2000) A note on the solution of conjugate heat transfer problems using SIMPLE-Like algorithms. Int J Heat Fluid Flow 21:463-467

27. Zhu J (1991) FAST-2D: a computer program for numerical simulation of two-dimensional incompressible flows with complex boundaries. Institute for Hydromechanics. University of Karlsruhe, Report No. 690 
28. Hardy TL, Curran FM (1987) Low power dc arcjet operation with hydrogen/nitrogen/ammonia mixtures. AIAA paper No. 87-1948

29. Bufton SA, Burton RL, Krier H (1995) Measured plasma properties at the exit plane of a $1 \mathrm{~kW}$ arcjet. AIAA Paper No. 95-3066

30. Liebeskind JG, Hanson RK, Cappelli MA (1993) Laser-induced fluorescence diagnostic for temperature and velocity measurements in a hydrogen arcjet plume. Appl Opt 32:6117-6127

31. Strom PV, Cappelli MA (1996) Stark broadening corrections to laser-induced fluorescence temperature measurements in a hydrogen arcjet plume. Appl Opt 35:4913-4918

32. Storm PV, Cappelli MA (1996) LIF characterization of arcjet nozzle flows. AIAA Paper No. 96-2987

33. Strom PV, Cappelli MA (1996) Radiative emission analysis of an expanding hydrogen arc plasma-II. Plume region diagnostics through radial emission. J Quant Spectrosc Radiat Transf 56:919-932

34. Cappelli MA, Liebeskind JG, Hanson RK, Butler GW, King DQ (1993) A comparison of arcjet plume properties to model predictions. AIAA Paper 93-0820

35. Takahashi Y, Kihara H, Abe K (2010) The effects of radiative heat transfer in arc-heated nonequilibrium flow simulation. J Phys D Appl Phys 43:185201 